\title{
Infinite sequences of almost symmetric non-Weierstrass numerical semigroups
}

\section{Jiryo Komeda ${ }^{1}$}

Received: 11 March 2021 / Accepted: 23 September 2021 / Published online: 11 October 2021

(c) The Author(s) 2021

\begin{abstract}
Let $u$ be any positive integer. We construct infinite sequences of almost symmetric non-Weierstrass numerical semigroups whose conductors are the genera double minus $2 u-1$. Moreover, let $v$ be any non-negative integer. We give an infinite number of non-Weierstrass numerical semigroups whose conductors are the genera double minus $2 v$.
\end{abstract}

Keywords Conductors of numerical semigroups - Almost symmetric numerical semigroups · Non-Weierstrass numerical semigroups · Pseudo-Frobenius numbers

\section{Introduction}

Let $\mathbb{N}_{0}$ be the additive monoid of non-negative integers. A submonoid $H$ of $\mathbb{N}_{0}$ is called a numerical semigroup if the complement $\mathbb{N}_{0} \backslash H$ is a finite set. The cardinality of $\mathbb{N}_{0} \backslash H$ is called the genus of $H$, denoted by $g(H)$. Let $(C, P)$ be a pointed curve of genus $g$ where a curve means a complete non-singular irreducible algebraic curve over an algebraically closed field $k$ of characteristic 0 in this article. We set

$$
H(P)=\left\{n \in \mathbb{N}_{0} \mid \text { there exists } f \in k(C) \text { such that }(f)_{\infty}=n P\right\}
$$

where $k(C)$ denotes the field of rational functions on $C$. Then $H(P)$ becomes a numerical semigroup of genus $g$. A numerical semigroup $H$ is said to be Weierstrass if there exists a pointed curve $(C, P)$ with $H(P)=H$. Hurwitz [2] posed the following

Communicated by Benjamin Steinberg.

This work was supported by JSPS KAKENHI Grant Number 18K03228.

$\triangle \quad$ Jiryo Komeda

komeda@gen.kanagawa-it.ac.jp

1 Department of Mathematics, Center for Basic Education and Integrated Learning, Kanagawa Institute of Technology, 1030 Shimo-Ogino, Atsugi, Kanagawa 243-0292, Japan 
problem in 1893: Is every numerical semigroup Weierstrass? This is a long-standing problem. In 1980 Buchweitz [1] gave a computable necessary condition for a numerical semigroup to be Weierstrass. Using this criterion he finally found an example of a non-Weierstrass numerical semigroup. About ten years later after Buchweitz gave the non-Weierstrass numerical semigroup, Torres [13] showed that the criterion given by Buchweitz is not a sufficient condition for a numerical semigroup to be Weierstrass. So the following problem is still unsolved:

Give a computable necessary and sufficient condition on a numerical semigroup to be Weierstrass.

Let $H$ be a numerical semigroup. We set $c(H)=\min \left\{c \in \mathbb{N}_{0} \mid c+\mathbb{N}_{0} \subseteq H\right\}$, which is called the conductor of $H$. The integer $c(H)-1$ is called the Frobenius number of $H$. We have $c(H) \leqq 2 g(H)$. A numerical semigroup $H$ is said to be symmetric if the equality $c(H)=2 g(H)$ holds. A numerical semigroup $H$ is said to be quasi-symmetric if the equality $c(H)=2 g(H)-1$ holds. We denote by $P F(H)$ the set

$$
\left\{f \in \mathbb{N}_{0} \backslash H \mid f+h \in H \text { for all } h \in H \text { with } h>0\right\} .
$$

Since $c(H)-1$ belongs to $P F(H)$, the set $P F(H)$ is non-empty. An element of $P F(H)$ is called a pseudo-Frobenius number of $H$. The cardinality of the set $P F(H)$ is called the type of $H$, which is denoted by $t(H)$. We have $c(H)+t(H) \leqq 2 g(H)+1$ (see Proposition 2.2 in [10]). A numerical semigroup $H$ is said to be almost symmetric if $c(H)+t(H)=2 g(H)+1$. Hence, any symmetric numerical semigroup is almost symmetric. Let $l$ be an integer with $l \geqq 2$. For any numerical semigroup $H$, we set

$$
d_{l}(H)=\left\{h^{\prime} \in \mathbb{N}_{0} \mid l h^{\prime} \in H\right\}
$$

which is also a numerical semigroup. In Sect. 2, using the result in Torres [13], we give infinite sequences

$$
H_{0,0}=H_{0} \stackrel{d_{2}}{\longleftarrow} H_{1} \stackrel{d_{2}}{\longleftarrow} H_{2} \stackrel{d_{2}}{\longleftarrow} \ldots \stackrel{d_{2}}{\longleftarrow} H_{i-1} \stackrel{d_{2}}{\longleftarrow} H_{i} \stackrel{d_{2}}{\longleftarrow} \ldots
$$

of symmetric non-Weierstrass numerical semigroups. In Sect. 3, for any positive integer $t$ and any almost symmetric numerical semigroup $H_{0}$ of type $t$, we construct infinite sequences

$$
H_{0} \stackrel{d_{3}}{\longleftarrow} H_{1} \stackrel{d_{3}}{\longleftarrow} H_{2} \stackrel{d_{3}}{\longleftarrow} \ldots \stackrel{d_{3}}{\longleftarrow} H_{i-1} \stackrel{d_{3}}{\longleftarrow} H_{i} \stackrel{d_{3}}{\longleftarrow} \ldots
$$

of almost symmetric numerical semigroups of type $t$, hence $c\left(H_{i}\right)=2 g\left(H_{i}\right)-(t-1)$. Oliveira and Stöhr [11] constructed quasi-symmetric non-Weierstrass numerical semigroups $H_{0,1}$. In Sect. 4 for any integer $u$ with $u \geqq 2$, we show that there are almost symmetric non-Weierstrass numerical semigroups $H_{0,2 u-1}$ of type $2 u$, hence $c\left(H_{0,2 u-1}\right)=2 g\left(H_{0,2 u-1}\right)-(2 u-1)$. Thereafter for any $u \geqq 1$, we construct infinite sequences of almost symmetric non-Weierstrass numerical semigroups whose starting 
points are the above numerical semigroups $H_{0,2 u-1}$ as follows:

$$
H_{0,2 u-1}=H_{0} \stackrel{d_{3}}{\longleftarrow} H_{1} \stackrel{d_{3}}{\longleftarrow} H_{2} \stackrel{d_{3}}{\longleftarrow} \ldots \stackrel{d_{3}}{\longleftarrow} H_{i-1} \stackrel{d_{3}}{\longleftarrow} H_{i} \stackrel{d_{3}}{\longleftarrow} \ldots
$$

with $c\left(H_{i}\right)=2 g\left(H_{i}\right)-(2 u-1)$. In Sect. 5 we study numerical semigroups whose conductors are even. Specifically, we give an infinite number of non-Weierstrass numerical semigroups $H$ with $c(H)=2 g(H)-2 v$ for any $v \geqq 1$.

\section{Symmetric non-Weierstrass numerical semigroups}

For a numerical semigroup $H$ we use the following notation: We set

$$
m=m(H)=\min \{h \in H \mid h>0\},
$$

which is called the multiplicity of $H$. Let

$$
s_{i}=\min \{h \in H \mid h \equiv i \bmod m\}
$$

for any $i$ with $1 \leqq i \leqq m-1$. The numerical semigroup $H$ is generated by $s_{1}, \ldots, s_{m-1}$. The set $\left\{m, s_{1}, \ldots, s_{m-1}\right\}$ is called the standard basis for $H$, which is denoted by $S(H)$. We set

$$
s_{\text {max }}=\max \left\{s_{i} \mid i=1,2, \ldots, m-1\right\} .
$$

We note that $c(H)=s_{\max }-m+1$. To construct infinite sequences of symmetric non-Weierstrass numerical semigroups we need the following lemma:

Lemma 1 Let $H$ be a symmetric numerical semigroup with multiplicity $m$ and $n$ be an odd number with $n \geqq c(H)+m-1$. We set

$$
\tilde{H}=2 H+n \mathbb{N}_{0}:=\left\{2 h+n l \mid h \in H, l \in \mathbb{N}_{0}\right\} .
$$

Then $\tilde{H}$ is a symmetric numerical semigroup whose genus is $2 g(H)+\frac{n-1}{2}$.

Proof Using Proposition 2.1 in [8] we get the description of $g(\tilde{H})$. We note that the proof of Proposition 2.1 in [8] works well even if $n \geqq c(H)+m-1$. Then we have

$$
c(\tilde{H})=n+2 s_{\max }-2 m+1=n-1+2\left(s_{\max }-m+1\right)=n-1+2 c(H) .
$$

Since $H$ is symmetric, we obtain

$$
c(\tilde{H})=n-1+4 g(H)=2 g(\tilde{H}),
$$

which implies that $\tilde{H}$ is symmetric. 
Remark 1 The semigroup $\tilde{H}$ in Lemma 1 is obtained by "gluing" and the Frobenius numbers are easily computed (see Theorem 9.2 and Proposition 9.11 in [12]). Using the above we can also prove that $\tilde{H}$ is symmetric.

The following is useful for constructing infinite sequence of non-Weierstrass numerical semigroups from a non-Weierstrass numerical semigroup:

Remark 2 Let $H$ be a non-Weierstrass numerical semigroup and $\tilde{H}$ be a numerical semigroup with $d_{2}(\tilde{H})=H$. If $g(\tilde{H}) \geqq 6 g(H)+1$, then $\tilde{H}$ is non-Weierstrass.

Proof Assume that $\tilde{H}$ is Weierstrass, i.e., there exists a pointed curve $(C, P)$ such that $H(P)=\tilde{H}$. If $g(\tilde{H}) \geqq 6 g(H)+4, g(\tilde{H})=6 g(H)+3$ or $6 g(H)+2$, and $g(\tilde{H})=6 g(H)+1$, by Corollary 2.10 in [13], Theorem B in [3] and Main Theorem in [9] respectively, $C$ is a double cover of some curve $C^{\prime}$ such that $P$ is its ramification point. Let $\pi: C \longrightarrow C^{\prime}$ be the double covering. Then we have $H=H(\pi(P))$ by Lemma 2 in [5], which implies that $H$ is Weierstrass. This is a contradiction.

Theorem 1 Let $H_{0}$ be a symmetric non-Weierstrass numerical semigroup. Then we have infinite sequences

$$
H_{0} \stackrel{d_{2}}{\longleftarrow} H_{1} \stackrel{d_{2}}{\longleftarrow} H_{2} \stackrel{d_{2}}{\longleftarrow} \ldots \stackrel{d_{2}}{\longleftarrow} H_{i-1} \stackrel{d_{2}}{\longleftarrow} H_{i} \stackrel{d_{2}}{\longleftarrow} \ldots
$$

of symmetric non-Weierstrass numerical semigroups.

Proof Let $H$ be a symmetric non-Weierstrass numerical semigroup with multiplicity $m$. For any odd $n \geqq c(H)+m-1$ the numerical semigroup $\tilde{H}=2 H+n \mathbb{N}_{0}$ is symmetric from Lemma 1 . If $n \geqq 8 g(H)+3$, then we have $g(\tilde{H})=2 g(H)+\frac{n-1}{2} \geqq$ $6 g(H)+1$. Hence by Remark 2 the numerical semigroup $\tilde{H}$ is non-Weierstrass. Repeating this process we get the desired infinite sequences.

\section{Infinite sequences of almost symmetric numerical semigroups}

Proposition 1 Let $H$ be a numerical semigroup with multiplicity $m$ and $n$ be an integer not divisible by 3 with

$$
n \geqq \max \{c(H)+m-1,3 m+1\} .
$$

Then we have $g\left(3 H+n \mathbb{N}_{0}\right)=3 g(H)+n-1$.

Proof Since $n \geqq 3 m, 3 H+n \mathbb{N}_{0}$ is a $3 m-$ semigroup. Moreover, the standard basis $S\left(3 H+n \mathbb{N}_{0}\right)$ is

$$
\begin{aligned}
& \left\{3 m, 3 s_{1}, \ldots, 3 s_{m-1}\right\} \cup\left\{n, n+3 s_{1}, \ldots, n+3 s_{m-1}\right\} \cup\left\{2 n, 2 n+3 s_{1},\right. \\
& \left.\quad \ldots, 2 n+3 s_{m-1}\right\},
\end{aligned}
$$

because $n \geqq c(H)+m-1=s_{\text {max }}$.

We put $\bar{H}_{1}=3 H+n \mathbb{N}_{0}$. We will count $\sharp\left(\mathbb{N} \backslash H_{1}\right)$. 
(a) For a non-negative integer $y$ we have $x=3 y \notin H_{1}$ if and only if $y \notin H$. We have $\sharp\left\{y \in \mathbb{N}_{0} \mid y \notin H\right\}=g(H)$.

(b) For an integer $y$ we have $x=n+3 y \in \mathbb{N}_{0} \backslash H_{1}$ if and only if $y \in \mathbb{N}_{0} \backslash H$ or $0<n+3 y<n$. We have

$$
\sharp\left\{y \in \mathbb{Z} \mid y \in \mathbb{N}_{0} \backslash H \text { or } 0<n+3 y<n\right\}=g(H)+\left[\frac{n}{3}\right] .
$$

(c) For an integer $y$ we have $x=2 n+3 y \in \mathbb{N}_{0} \backslash H_{1}$ if and only if $y \in \mathbb{N}_{0} \backslash H$ or $0<2 n+3 y<2 n$. We have

$$
\sharp\left\{y \in \mathbb{Z} \mid y \in \mathbb{N}_{0} \backslash H \text { or } 0<2 n+3 y<2 n\right\}=g(H)+\left[\frac{2 n}{3}\right] .
$$

Thus, we obtain

$$
g\left(H_{1}\right)=\sharp\left(\mathbb{N}_{0} \backslash H_{1}\right)=3 g(H)+\left[\frac{n}{3}\right]+\left[\frac{2 n}{3}\right]=3 g(H)+n-1 .
$$

We will show from now on that there is an infinite sequence of almost symmetric numerical semigroups whose starting point is any almost symmetric numerical semigroup. For that purpose we need to check whether a given numerical semigroup is almost symmetric. To show that a numerical semigroup $H$ is almost symmetric we investigate the set $P F(H)$ of pseudo-Frobenius numbers. The following lemma is useful for describing the set $P F(H)$ :

Lemma 2 Let $H$ be a numerical semigroup with multiplicity $m$. Then we have

$$
P F(H)=\left\{s-m \mid s \in S P F_{+}(H)\right\}
$$

where we set

$$
S P F_{+}(H)=\left\{s \in S(H) \mid s+s^{\prime} \notin S(H) \text { for all } s^{\prime} \in S(H)\right\}
$$

Proof Let $s \in S P F_{+}(H)$. Take $h \in H$ with $h>0$. Then $h=s^{\prime}+l m$ for some $s^{\prime} \in S(H)$ with $l \in \mathbb{N}_{0}$. We have

$$
s-m+h=s+s^{\prime}+(l-1) m=s^{\prime \prime}+l^{\prime} m+(l-1) m=s^{\prime \prime}+\left(l^{\prime}+l-1\right) m
$$

for some $s^{\prime \prime} \in S(H)$ with a positive integer $l^{\prime}$. Hence we get $s-m+h \in H$. Thus, we have $s-m \in P F(H)$.

Let $f \in P F(H)$. Then we have $f=s-m$ with $s \in S(H)$, because $f \notin H$ and $f+m \in H$. Moreover, we obtain $H \ni f+s^{\prime}=s-m+s^{\prime}$ for any $s^{\prime} \in S(H)$, which implies that $s+s^{\prime}=h+m$ with $h \in H$. Hence, we get $s+s^{\prime} \notin S(H)$. 
We will show how to construct an almost symmetric numerical semigroup $\tilde{H}$ from any almost symmetric numerical semigroup $H$ satisfying $d_{3}(\tilde{H})=H$. We denote by $\left\langle a_{1}, \ldots, a_{l}\right\rangle$ the additive monoid generated by positive integers $a_{1}, \ldots, a_{l}$. This notation is useful for describing a numerical semigroup.

Theorem 2 Let $t$ be a positive integer. Let $H$ be an almost symmetric numerical semigroup with multiplicity $m$ and $c(H)=2 g(H)-(t-1)$. Let $n$ be an integer with $n \geqq \max \{c(H)+m-1,3 m+1\}$ which is not divisible by 3 . We set

$$
\tilde{H}=3 H+\left\langle n, 2 n+3 f_{1}, \ldots, 2 n+3 f_{t-1}\right\rangle
$$

where $P F^{*}(H)=P F(H) \backslash\{c(H)-1\}=\left\{f_{1}, \ldots, f_{t-1}\right\}$, because $H$ is almost symmetric. Then we have

$$
g(\tilde{H})=3 g(H)+n-1-(t-1) \text { and } c(\tilde{H})=2 g(\tilde{H})-(t-1) .
$$

Moreover, we obtain $t(\tilde{H})=t$, which implies that the numerical semigroup $\tilde{H}$ is almost symmetric.

Proof It follows from Lemma 2 that

$$
\begin{aligned}
& S(\tilde{H})=\left\{3 m, 3 s_{1}, \ldots, 3 s_{m-1}, n, n+3 s_{1}, \ldots, n+3 s_{m-1}\right\} \\
& \quad \cup\left\{2 n+3 f_{1}, \ldots, 2 n+3 f_{t-1}\right\} \cup\{2 n+3 s \mid s \in S(H) \text { with } s-m \notin P F(H)\} .
\end{aligned}
$$

Using Proposition 1 and $f_{i} \in P F(H)$ for any $i$ with $1 \leqq i \leqq t-1$, we get

$$
g(\tilde{H})=3 g(H)+n-1-(t-1)
$$

Since $2 n+3 s_{\max }$ is the maximum element in $S(\tilde{H})$, we have $2 n+3 s_{\max }-3 m \in$ $P F(\tilde{H})$. Moreover, we have

$$
\begin{aligned}
& c(\tilde{H})=2 n+3 s_{\max }-3 m+1=3\left(s_{\max }-m+1\right)+2(n-1) \\
& \quad=3 c(H)+2(n-1) \\
& \quad=3(2 g(H)-(t-1))+2(n-1) \\
& \quad=2(3 g(H)+n-t)-t+1=2 g(\tilde{H})-(t-1) .
\end{aligned}
$$

We set $s^{(i)}=f_{i}+m$ for all $i$. Then

$$
\left(n+3 s^{(i)}\right)+n=\left(2 n+3 f_{i}\right)+3 m \notin S(\tilde{H}) .
$$

Moreover, for any $s \in S(H)$ we have

$$
\left(n+3 s^{(i)}\right)+3 s=n+3\left(s^{(i)}+s\right)=n+3\left(s^{\prime}+v m\right)
$$

with a positive integer $v$ from Lemma 2, because $f_{i} \in P F(H)$. Using Lemma 2 again we obtain $n+3 f_{i} \in P F(\tilde{H})$ for all $i=1, \ldots, t-1$. Thus, we have $t(\tilde{H}) \geqq t$. 
Moreover, the equality $c(\tilde{H})=2 g(\tilde{H})-(t-1)$ implies that $t(\tilde{H}) \leqq t$. Hence, we get $t(\tilde{H})=t$. Thus, $\tilde{H}$ is almost symmetric.

Here, we give an example of an almost symmetric numerical semigroup $H$ with $c(H)=2 g(H)-(t-1)$ for any positive integer $t$.

Example 1 Let $t$ be a positive integer. We set $m=t+1$. Let $H=\langle m, m+1, \ldots, m+$ $m-1\rangle$. By Lemma 2 we have $t(H)=m-1=t$. Moreover, we obtain

$$
c(H)=m=2(m-1)-(m-2)=2 g(H)-(t-1) .
$$

Hence, the numerical semigroup $H$ is almost symmetric.

Using Theorem 2 repeatedly we get infinite sequences of almost symmetric numerical semigroups.

Corollary 1 Let $t$ be a positive integer. Let $H$ be any almost symmetric numerical semigroup with $c(H)=2 g(H)-(t-1)$. Then there exists an infinite sequences

$$
H_{0}=H \stackrel{d_{3}}{\longleftarrow} H_{1} \stackrel{d_{3}}{\longleftarrow} H_{2} \stackrel{d_{3}}{\longleftarrow} \ldots \stackrel{d_{3}}{\longleftarrow} H_{i-1} \stackrel{d_{3}}{\longleftarrow} H_{i} \stackrel{d_{3}}{\longleftarrow} \ldots
$$

of almost symmetric numerical semigroups whose conductors are equal to the genera double minus $t-1$.

\section{Almost symmetric non-Weierstrass numerical semigroups with odd conductor}

To find almost symmetric non-Weierstrass numerical semigroups with odd conductor we use the construction in the following lemma whose origin is Scholium 3.5 in [13] and Theorem 5.1 in [11].

Lemma 3 Let $u$ be a positive integer and $H$ be a numerical semigroup with multiplicity $m \geqq 2$. Let $g$ be an integer with $g \not \equiv u \bmod 3$ and

$$
g>\max \{4 u-3,2 u+2 c(H)+m-4\} .
$$

We set

$$
\tilde{H}=3 H \cup\left(g+2 u+3 \mathbb{N}_{0}\right) \cup\{2 g-2 u-3 r \mid r \in \mathbb{Z} \backslash H\} .
$$

Then the following hold:

(i) $\tilde{H}$ is a numerical semigroup of genus $g$.

(ii) We have $c(\tilde{H})=2 g-(2 u-1)$, hence the maximum element in $S(\tilde{H})$ is $2 g-$ $2 u+3 m$. 
(iii) We obtain

$P F(\tilde{H}) \subseteq\{g+2 u+3 l-3 m \mid l=0,1, \ldots, m-1\} \cup\{2 g-2 u+3 m-3 m\}$, which implies that $t(\tilde{H}) \leqq m+1$.

Proof (i) First, we will prove that $\tilde{H}$ becomes a submonoid of the additive monoid $\mathbb{N}_{0}$. We set

$$
A=3 H, B=g+2 u+3 \mathbb{N}_{0} \text { and } C=\{2 g-2 u-3 r \mid r \in \mathbb{Z} \backslash H\}
$$

We note that the minimum element of $C$ is $2 g-2 u-3(c(H)-1)$, which is non-negative, because we have

$$
\begin{aligned}
2 g-2 u-3(c(H)-1) & \geqq 2(2 u+2 c(H)+m-3)-2 u-3(c(H)-1) \\
& =2 u+c(H)+2 m-3 \geqq 2 u+c(H)+1>0 .
\end{aligned}
$$

It is trivial that

$$
A+A \subseteq A, A+B \subseteq B \text { and } A+C \subseteq C
$$

Let $l \in \mathbb{N}_{0}$ and $l^{\prime} \in \mathbb{N}_{0}$. We have

$$
\begin{aligned}
(g+2 u+3 l)+\left(g+2 u+3 l^{\prime}\right) & =2 g-2 u+6 u+3\left(l+l^{\prime}\right) \\
& =2 g-2 u-3\left(-2 u-l-l^{\prime}\right),
\end{aligned}
$$

which implies that $B+B \subseteq C$, because $-2 u-l-l^{\prime}<0$.

Let $l \in \mathbb{N}_{0}$ and $r \in \mathbb{Z} \backslash H$. We have

$$
(g+2 u+3 l)+(2 g-2 u-3 r)=3(g+l-r) .
$$

Since $g \geqq 2 u+2 c(H)+m-3>2 u+2 c(H)-2$, we get

$$
\begin{aligned}
g+l-r & \geqq g-r>2 u+2 c(H)-2-r \geqq 2+2 c(H)-2-r \\
& =2 c(H)-r \geqq 2 c(H)-(c(H)-1)=c(H)+1,
\end{aligned}
$$

because $r \leqq c(H)-1$. Hence, we get $g+l-r \in H$, which implies that $B+C \subseteq A$.

Let $r \in \mathbb{Z} \backslash H$ and $r^{\prime} \in \mathbb{Z} \backslash H$. Then we have

$$
(2 g-2 u-3 r)+\left(2 g-2 u-3 r^{\prime}\right)=g+2 u+3\left(g-2 u-r-r^{\prime}\right) .
$$

It follows from $g>\max \{4 u-3,2 u+2 c(H)+m-4\}$ that

$$
\begin{aligned}
g-2 u-r-r^{\prime} & \geqq g-2 u+2-2 c(H) \\
& \geqq 2 u+2 c(H)+m-3-2 u+2-2 c(H)=m-1>0,
\end{aligned}
$$


which implies that $C+C \subseteq B$. Thus, $\tilde{H}$ becomes a submonoid of $\mathbb{N}_{0}$. By the definition of $\tilde{H}$ it is a numerical semigroup.

Secondly, we will show that the genus of the numerical semigroiup $\tilde{H}$ is equal to $g$. We have

$$
\begin{aligned}
\mathbb{N}_{0} \backslash \tilde{H}= & 3 \mathbb{N}_{0} \backslash 3 H \cup\left(3 \mathbb{N}_{0}+a\right) \backslash\left\{g+2 u+3 \mathbb{N}_{0}\right\} \\
& \cup\left(3 \mathbb{N}_{0}+b\right) \backslash\{2 g-2 u-3 r \mid r \in \mathbb{Z} \backslash H\}
\end{aligned}
$$

where $g+2 u \equiv a \bmod 3$ and $2 g-2 u \equiv b \bmod 3$ with $\{a, b\}=\{1,2\}$, because $g \not \equiv u \bmod 3$. We obtain that

$$
\sharp\left(3 \mathbb{N}_{0} \backslash 3 H\right)=g(H) \text { and } \sharp\left(\left(3 \mathbb{N}_{0}+a\right) \backslash\left\{g+2 u+3 \mathbb{N}_{0}\right\}\right)=\frac{g+2 u-a}{3} .
$$

Moreover, the maximum element in $\left(3 \mathbb{N}_{0}+s\right) \backslash\{2 g-2 u-3 r \mid r \in \mathbb{Z} \backslash H\}$ is $2 g-2 u$, which implies that

$$
\sharp\left(\left(3 \mathbb{N}_{0}+b\right) \backslash\{2 g-2 u-3 r \mid r \in \mathbb{Z} \backslash H\}\right)=\frac{2 g-2 u+3-b}{3}-g(H) .
$$

Combining the above results about the cardinalities of the three sets we get

$$
g(\tilde{H})=\sharp\left(\mathbb{N}_{0} \backslash \tilde{H}\right)=\frac{3 g-a-b+3}{3}=g .
$$

(ii) We have $3(c(H)-1) \notin 3 H$ and $3 l \in 3 H$ for any $l \geqq c(H)$. Moreover, it is clear that $g+2 u-3 \notin g+2 u+3 \mathbb{N}_{0}$ and $g+2 u+3 l \in g+2 u+3 \mathbb{N}_{0}$ for all $l \geqq 0$. It follows that $2 g-2 u \notin C$ and $2 g-2 u+3 l^{\prime} \in C$ for all $l^{\prime}>0$. Since we have the inequalities

$$
\begin{aligned}
& 2 g-2 u-(g+2 u-3)=g-(4 u-3)>0 \text { and } \\
& 2 g-2 u-(3 c(H)-3)=2\left(g-\left(u+\frac{3}{2}(c(H)-1)\right)\right. \\
& \geqq 2\left(2 u+2 c(H)+m-3-u-\frac{3}{2}(c(H)-1)\right) \\
& \quad=2\left(u+\frac{1}{2} c(H)+m-\frac{3}{2}\right)>0,
\end{aligned}
$$

we obtain $c(\tilde{H})=2 g-(2 u-1)$. Hence, the maximal element in $S(\tilde{H})$ is equal to

$$
c(\tilde{H})-1+3 m=2 g-(2 u-1)-1+3 m=2 g-2 u+3 m .
$$


(iii) We obtain

$$
\begin{aligned}
S(\tilde{H})= & \left\{3 m, 3 s_{1}, \ldots, 3 s_{m-1}\right\} \cup\{g+2 u+3 l \mid l=0,1, \ldots, m-1\} \\
& \cup\left\{2 g-2 u-3\left(s_{i}-m\right) \mid i=1, \ldots, m-1\right\} \cup\{2 g-2 u+3 m\} .
\end{aligned}
$$

Moreover, we have

$$
3 s_{i}+2 g-2 u-3\left(s_{i}-m\right)=2 g-2 u+3 m
$$

for all $i=1, \ldots, m-1$. By Lemma 2 we get the inclusion in (iii).

Proposition 2 Let $u, H, g$ and $\tilde{H}$ be as in Lemma 3. Moreover, assume that $m \leqq 2 u-1$. Then we get $t(\tilde{H})=m+1$. If $m=2 u-1$, then $\tilde{H}$ is an almost symmetric numerical semigroup with $c(\tilde{H})=2 g(\tilde{H})-(2 u-1)$.

Proof For $l \geqq 0$ we have

$(g+2 u+3 l)+3 s_{i} \geqq g+2 u+3 s_{i} \geqq g+2 u+3(m+1)>g+2 u+3(m-1)$.

Moreover, we obtain

$$
\begin{aligned}
& \left((g+2 u+3 l)+\left(2 g-2 u-3\left(s_{i}-m\right)\right)\right)-3 s_{\max } \geqq 3 g-3 s_{i}+3 m-3 s_{\max } \\
& \geqq 3(2 u+2 c(H)+m-3)-3 s_{i}+3 m-3 s_{\max } \\
& \geqq 3(2 u+2 c(H)-1)-3 s_{i}+3 m-3 s_{\max } \\
& \quad=3\left(2 u+c(H)-s_{\max }+m-1+c(H)-s_{i}\right)=3\left(2 u+c(H)-s_{i}\right) \\
& =3\left(2 u+s_{\max }-m+1-s_{i}\right) \\
& \geqq 3(2 u-m+1) \geqq 3(2 u-(2 u-1)+1)=6>0,
\end{aligned}
$$

because $m \leqq 2 u-1$.

Lastly, for $l \geqq 0$ and $l^{\prime} \geqq 0$ we get

$$
\begin{aligned}
& (g+2 u+3 l)+\left(g+2 u+3 l^{\prime}\right)-(2 g-2 u+3 m) \\
& \quad \geqq 2 g+4 u-(2 g-2 u+3 m)=3(2 u-m) \geqq 3>0
\end{aligned}
$$

by the assumption $m \leqq 2 u-1$. By Lemmas 2 and 3(iii) we obtain

$$
P F(\tilde{H})=\{g+2 u+3 l-3 m \mid l=0,1, \ldots, m-1\} \cup\{2 g-2 u+3 m-3 m\} .
$$

Thus, we get $t(\tilde{H})=m+1$.

If $m=2 u-1$, then it follows from Lemma 3(ii) that

$$
\begin{aligned}
& c(\tilde{H})+t(\tilde{H})=2 g(\tilde{H})-(2 u-1)+m+1 \\
& \quad=2 g(\tilde{H})-(2 u-1)+(2 u-1)+1=2 g(\tilde{H})+1,
\end{aligned}
$$

which implies that $\tilde{H}$ is almost symmetric. 
The following result is useful for getting non-Weierstrass numerical semigroups:

Remark 3 Let $H$ be a numerical semigroup with $g(H) \geqq 15 g\left(d_{3}(H)\right)+11$. If $H$ is Weierstrass, then so is $d_{3}(H)$.

Proof By Corollaries 2.6 and 2.7 in [14] we can apply to $H$ the proof of Theorem 5.1 in [11]. Hence we can see that $d_{3}(H)$ is Weierstrass.

We find almost symmetric non-Weierstrass numerical semigroups $\tilde{H}$ with an odd conductor $c(\tilde{H})=2 g(\tilde{H})-(2 u-1)$ for any positive integer $u$ using Proposition 2 .

Theorem 3 Let $u \geqq 7$. Then there exists an almost symmetric non-Weierstrass numerical semigroup $\tilde{H}$ with $c(\tilde{H})=2 g(\tilde{H})-(2 u-1)$.

Proof Let $m$ be an odd number $\geqq 13$. We set $m=2 u-1$, which implies that $u=$ $\frac{m+1}{2} \geqq 7$. Then there exists a non-Weierstrass $m$-semigroup $H$ (for example, see [6]). Let $g$ be an integer as in Lemma 3. Moreover, we assume that $g \geqq 15 g(H)+11$. We set

$$
\tilde{H}=3 H \cup\left(g+2 u+3 \mathbb{N}_{0}\right) \cup\{2 g-2 u-3 r \mid r \in \mathbb{Z} \backslash H\} .
$$

By Remark 3 the numerical semigroup $\tilde{H}$ is non-Weierstrass, because so is $H=$ $d_{3}(\tilde{H})$. Moreover, it follows from Proposition 2 that $\tilde{H}$ is an almost symmetric numerical semigroup with $c(\tilde{H})=2 g(\tilde{H})-(2 u-1)$.

Theorem 4 Let $u=3,5$ or 6 . Then there exists an almost symmetric non-Weierstrass numerical semigroup $\tilde{H}$ with $c(\tilde{H})=2 g(\tilde{H})-(2 u-1)$.

Proof We set $m=2 u+2$. Let $H, g$ and $\tilde{H}$ be as in Lemma 3. By Lemma 3(iii) we have

$$
P F(\tilde{H}) \subseteq\{g+2 u+3 l-3 m \mid l=0,1, \ldots, m-1\} \cup\{2 g-2 u+3 m-3 m\} .
$$

We obtain

$$
\begin{aligned}
& (g+2 u)+(g+2 u+3 \cdot 2)=(g+2 u+3 \cdot 1)+(g+2 u+3 \cdot 1) \\
& \quad=2 g-2 u+3(2 u+2)=2 g-2 u+3 m
\end{aligned}
$$

from $m=2 u+2$. Hence, by Lemma 2 we get

$$
P F(\tilde{H}) \subseteq\{g+2 u+3 l-3 m \mid l=3, \ldots, m-1\} \cup\{2 g-2 u+3 m-3 m\}
$$

We have

$$
(g+2 u+3 l)+3 s_{i}>g+2 u+3(m-1)
$$


for $l \geqq 0$. Moreover, for $l \geqq 3$ we obtain

$$
\begin{aligned}
& \left((g+2 u+3 l)+\left(2 g-2 u-3\left(s_{i}-m\right)\right)\right)-3 s_{\max } \geqq 3 g+9+3 m-3\left(s_{i}+s_{\max }\right) \\
& \quad \geqq 3(2 u+2 c(H)+m-3)+9+3 m-3\left(s_{i}+s_{\text {max }}\right) \\
& \quad=3(2 u-m+1)+9-9-3+6 c(H)+9 m-3\left(s_{i}+s_{\max }\right) \\
& \quad=3(2 u-m+1)+9+6(c(H)-1+m)-3\left(s_{i}+s_{\max }\right)-3+3 m-3 \\
& \quad \geqq 3(2 u-m+1)+9+3(m-2) \geqq 3(m-2-m+1)+9=6>0
\end{aligned}
$$

from $m=2 u+2$. Lastly, for $l \geqq 3$ and $l^{\prime} \geqq 0$ we get

$$
\begin{aligned}
& (g+2 u+3 l)+\left(g+2 u+3 l^{\prime}\right)-(2 g-2 u+3 m) \\
& \quad \geq 2 g+4 u-(2 g-2 u+3 m)+9=3(2 u-m)+9 \\
& \quad=3(m-2-m)+9=3>0
\end{aligned}
$$

from $m=2 u+2$. Thus, we obtain

$$
P F(\tilde{H})=\{g+2 u+3 l-3 m \mid l=3, \ldots, m-1\} \cup\{2 g-2 u+3 m-3 m\},
$$

which implies that $t(\tilde{H})=m-2=2 u$. Since we have $c(\tilde{H})=2 g(\tilde{H})-(2 u-1)$ from Lemma 3(ii), the numerical semigroup $\tilde{H}$ is almost symmetric.

For $m=8,12,14$, i.e., $u=3,5,6$, respectively there exists a non-Weierstrass $m$-semigroup from [6,7]. Assume that $g \geqq 15 g(H)+11$. By Remark 3 the numerical semigroup $\tilde{H}$ is non-Weierstrass.

Theorem 5 There exists an almost symmetric non-Weierstrass numerical semigroup $\tilde{H}$ with $c(\tilde{H})=2 g(\tilde{H})-7$.

Proof Let $u=4$ and $m=2 u=8$. Let $H=2\langle 4,6,9,11\rangle+\langle n, n+4\rangle$ where $n$ is an odd integer with $n \geqq 45$. Let $g$ and $\tilde{H}$ be as in Lemma 3. By Lemma 3(iii) we have

$$
P H(\tilde{H}) \subseteq\{g+8+3 l-24 \mid l=0,1, \ldots, 7\} \cup\{2 g-8+24-24\} .
$$

It follows from $m=2 u=8$ that $g+8+g+8=2 g-8+24$. Hence, we get

$$
P H(\tilde{H}) \subseteq\{g+8+3 l-24 \mid l=1, \ldots, 7\} \cup\{2 g-8+24-24\} .
$$

We obtain

$$
\begin{aligned}
& (g+2 u)+\left(2 g-2 u-3\left(s_{i}-m\right)\right)-3 s_{\text {max }} \\
& \geqq 3(2 u+2 c(H)+5)-3 s_{i}+3 m-3 s_{\text {max }}>6 u+6 c(H)+3 m-6 s_{\text {max }} \\
& \quad=6 u+6 c(H)+3 m-6(c(H)+m-1)=6 u-3 m+6=24-24+6>0
\end{aligned}
$$

and

$$
(g+2 u+3 l)+3 s_{i}>g+2 u+3(m-1) .
$$


Moreover, for any $l \geqq 1$ and $l^{\prime} \geqq 0$ we have

$$
\begin{aligned}
& (g+2 u+3 l)+\left(g+2 u+3 l^{\prime}\right)-(2 g-2 u+3 m) \\
& \quad \geqq 2 g+4 u-(2 g-2 u+3 m)+3=3(2 u-m)+3=3>0
\end{aligned}
$$

from $m=2 u$.

Thus, we obtain

$$
P H(\tilde{H})=\{g+8+3 l-24 \mid l=1, \ldots, 7\} \cup\{2 g-8+24-24\},
$$

which implies that $t(\tilde{H})=8$. By Lemma 3 we have $c(\tilde{H})=2 g(\tilde{H})-7$. Hence, $\tilde{H}$ is almost symmetric. By $[7,9,13]$ the numerical semigroup $H$ is non-Weierstrass. Assum that $g \geqq 15 g(H)+11$. Since $d_{3}(\tilde{H})=H$, it follows from Remark 3 that the numerical semigroup $\tilde{H}$ is non-Weierstrass.

Theorem 6 There exists an almost symmetric non-Weierstrass numerical semigroup $\tilde{H}$ with $c(\tilde{H})=2 g(\tilde{H})-3$.

Proof Let $u=2$ and $m=2 u+4=8$. Let $H=2\langle 4,6,9,11\rangle+\langle n, n+4\rangle$ where $n$ is an odd integer with $n \geqq 45$. Let $g$ and $\tilde{H}$ be as in Lemma 3. By Lemma 3 we have

$$
P H(\tilde{H}) \subseteq\{g+4+3 l-24 \mid l=0,1, \ldots, 7\} \cup\{2 g-4+24-24\}
$$

We get

$$
\begin{aligned}
(g+2 u)+(g+2 u+3 \cdot 4) & =(g+2 u+3 \cdot 1)+(g+2 u+3 \cdot 3) \\
& =(g+2 u+3 \cdot 2)+(g+2 u+3 \cdot 2) \\
& =2 g+4 u+12=2 g+8+12=2 g-4+24,
\end{aligned}
$$

which implies that

$$
P H(\tilde{H}) \subseteq\{g+4+3 l-24 \mid l=5,6,7\} \cup\{2 g-4+24-24\} .
$$

We obtain

$$
\begin{aligned}
& (g+2 u)+\left(2 g-2 u-3\left(s_{i}-m\right)\right)-3 s_{\max } \\
& \geq 3(2 u+2 c(H)+5)-3 s_{i}+3 m-3 s_{\max } \\
& \quad=3\left(2 u+2 s_{\max }-2 m+2+5\right)-3 s_{i}+3 m-3 s_{\max } \\
& >6 u-3 m+21=12-24+21=9>0
\end{aligned}
$$

and

$$
(g+2 u+3 l)+3 s_{i}>g+2 u+3(m-1) .
$$


Moreover, for any $l \geqq 5$ and $l^{\prime} \geqq 0$ we have

$$
\begin{aligned}
& (g+2 u+3 l)+\left(g+2 u+3 l^{\prime}\right)-(2 g-2 u+3 m) \\
& \quad \geqq 2 g+4 u-(2 g-2 u+3 m)+15 \\
& \quad=3(2 u-m)+15=3(2 u-2 u-4)+15=3>0
\end{aligned}
$$

from $m=2 u+4$. Hence, we obtain

$$
P H(\tilde{H})=\{g+4+3 l-24 \mid l=5,6,7\} \cup\{2 g-4+24-24\},
$$

which implies that $t(\tilde{H})=4$. By Lemma 3 we have $c(\tilde{H})=2 g(\tilde{H})-3$. Hence, $\tilde{H}$ is almost symmetric. Assume that $g \geqq 15 g(H)+11$. By the same way as the proof of Theorem 5 the numerical semigroup $\tilde{H}$ is non-Weierstrass.

By Theorem 5.1 in [11] we obtain the following:

Remark 4 There exists a quasi-symmetric non-Weierstrass numerical semigroup $\tilde{H}$, i.e., $c(\tilde{H})=2 g(\tilde{H})-1$.

By Theorems 3, 4, 5, 6 and Remark 4 we obtain the following:

Theorem 7 For any positive integer $u$ there exists an almost symmetric nonWeierstrass numerical semigroup $\tilde{H}$ with $c(\tilde{H})=2 g(\tilde{H})-(2 u-1)$.

By Theorems 2, 7 and [11] we obtain the following:

Corollary 2 For any $u \geqq 1$ we have infinite sequences

$$
\tilde{H}=H_{0} \stackrel{d_{3}}{\longleftarrow} H_{1} \stackrel{d_{3}}{\longleftarrow} H_{2} \stackrel{d_{3}}{\longleftarrow} \ldots \stackrel{d_{3}}{\longleftarrow} H_{i-1} \stackrel{d_{3}}{\longleftarrow} H_{i} \stackrel{d_{3}}{\longleftarrow} \ldots
$$

of almost symmetric non-Weierstrass numerical semigroups whose conductors are genera double minus $2 u-1$.

\section{Non-Weierstrass numerical semigroups with even conductor}

First, we give non-Weierstrass numerical semigroups $\tilde{H}$ with $c(\tilde{H})=2 g(\tilde{H})-2 v$ where $v$ is an odd number in the following Proposition:

Proposition 3 Let $m$ and $l$ be integers larger than 1. We set

$$
\begin{aligned}
& H=\langle 2 m, 2 m+2 \cdot 1, \ldots, 2 m+2 \cdot(m-1), 2 l m+2 \cdot 0+1, \\
& \ldots, 2 l m+2 \cdot(m-1)+1\rangle .
\end{aligned}
$$

For any odd integer $n$ with $n \geqq 2 c(H)+2 m-1$ we set $\tilde{H}=2 H+\langle n, n+4\rangle$.

(i) We have $g(H)=m(l+1)-1, c(H)=2 g(H)-2(m-1)$ and $t(H)=2 m-1$, hence $H$ is almost symmetric. 
(ii) We have $g(\tilde{H})=2 g(H)+\frac{n-1}{2}-1, c(\tilde{H})=2 g(\tilde{H})-2(2 m-3)$ and $t(\tilde{H})=2 m-1$.

(iii) If $n \geqq 8 g(H)+11$, then $\tilde{H}$ is non-Weierstrass.

Proof (i) We have

$$
g(H)=m-1+\operatorname{lm}=m(l+1)-1
$$

and

$$
c(H)=2 l m+2 \cdot(m-1)+1-2 m+1=2 l m=2 g(H)-2(m-1) .
$$

Moreover, it follows from Lemma 2 that $t(H)=2 m-1$.

(ii) Since $2=2 m+2 \cdot 1-2 m$, the genus $g(\tilde{H})$ of $\tilde{H}$ equals to $2 g(H)+\frac{n-1}{2}-1$.

We have

$$
\begin{aligned}
c(\tilde{H}) & =n+2(2 l m+2 \cdot(m-1)+1)-4 m+1=n+4 l m-1 \\
& =2\left(2(m(l+1)-1)+\frac{n-1}{2}-1\right)-2(2 m-3)=2 g(\tilde{H})-2(2 m-3) .
\end{aligned}
$$

Moreover, we have $t(\tilde{H})=2 m-1$. As a matter of fact, we obtain that

$$
(n+4)+2(2 l m+1)=n+2(2 l m+2 \cdot 1+1) \in S(\tilde{H}),
$$

which implies that $n+4-4 m \notin P F(\tilde{H})$. Hence, the set $P F(\tilde{H})$ is equal to

$$
\begin{aligned}
& \left\{2(2 m+2)-4 m, n+2 s_{1}-4 m, n+2 s_{3}-4 m, n+2 s_{4}-4 m,\right. \\
& \left.\quad \ldots, n+2 s_{2 m-1}-4 m\right\}
\end{aligned}
$$

where $S(H)=\left\{2 m, s_{1}, s_{2}, s_{3}, \ldots, s_{2 m-1}\right\}$ is the standard basis for $H$, i.e., $s_{2 i}=$ $2 m+2 i$ for $i=1, \ldots, m-1$ and $s_{2 i+1}=2 l m+2 i+1$ for $i=0,1, \ldots, m-1$.

(iii) We will show that the numerical semigroup $\tilde{H}$ is non-Weierstrass. Assume that $\tilde{H}$ is Weierstrass. Since we have

$g(\tilde{H})=2 g(H)+\frac{n-1}{2}-1 \geqq 2 g(H)+\frac{8 g(H)+11-1}{2}-1=6 g(H)+4$,

it follows from [13] that there is a double covering $\pi: \tilde{C} \longrightarrow C$ with a ramification point $\tilde{P}$ over $P$ such that $H(\tilde{P})=\tilde{H}$ and $H(P)=H$. Moreover, there is a divisor $D=\frac{n+1}{2} P-Q$ with a point $Q$ of $C$ distinct form $P$ such that $h^{0}(2 P+Q)=2$. Thus, $C$ is a trigonal curve with the point $P$ with

$$
\begin{aligned}
H(P)= & \langle 2 m, 2 m+2 \cdot 1, \ldots, 2 m+2 \cdot(m-1), 2 l m \\
& +2 \cdot 0+1, \ldots, 2 l m+2 \cdot(m-1)+1\rangle .
\end{aligned}
$$


By Theorem on the page 172 in [4] we must have

$$
\begin{aligned}
\mathbb{N}_{0} \backslash H & =\{1,2, \ldots, 2 m-1,2 m+1,2(m+1)+1, \ldots, 2(m l-1)+1\} \\
& =\{1,2, \ldots, 2 n+1,2(n+1)+1, \ldots, 2(g-n-1)+1\}
\end{aligned}
$$

with $\frac{g-1}{3} \leqq n \leqq \frac{g}{2}$ where we set $m=n+1$. Here, we note that $g=g(H)=$ $m(l+1)-1$ and $g-n-1=m l-1$. We must have

$$
m-1=n \geqq \frac{g-1}{3}=\frac{m(l+1)-1-1}{3},
$$

which implies that $l \leqq 2-\frac{1}{m}<2$. This contradicts $l \geqq 2$. Thus, $\tilde{H}$ should be non-Weierstrass.

We prove the following lemma which is used at the construction of non-Weierstrass numerical semigroups $\tilde{H}$ with $c(\tilde{H})=2 g(\tilde{H})-2 v$ where $v$ is an even number:

Lemma 4 Let $v$ be a positive integer. Let $H$ be a numerical semigroup of type $t$ with multiplicity $m$ and $c(H)=2 g(H)-2 v$. We take a subset $\left\{f_{1}, \ldots, f_{q}\right\}$ of $P F^{*}(H)=$ $P F(H) \backslash\{c(H)-1\}$ with $0 \leqq q \leqq t-1$. For an odd integer $n$ with $n \geqq 2 c(H)+2 m-1$ we set

$$
\tilde{H}=2 H+\left\langle n, n+2 f_{1}, \ldots, n+2 f_{q}\right\rangle .
$$

Then we have $c(\tilde{H})=2 g(\tilde{H})-2(v+v-q)$ and $t(\tilde{H}) \geqq t-q$.

Proof Since we have $P F^{*}(H) \supseteq\left\{f_{1}, \ldots, f_{q}\right\}$, we get

$$
g(\tilde{H})=2 g(H)+\frac{n-1}{2}-q
$$

and

$$
\begin{aligned}
c(\tilde{H})= & n+2 s_{\text {max }}-2 m+1=2\left(s_{\max }-m+1\right)+n-1=2 c(H)+n-1 \\
= & 2(2 g(H)-2 v)+n-1=2\left(2 g(H)+\frac{n-1}{2}-q\right) \\
& -4 v+2 q=2 g(\tilde{H})-2(v+v-q) .
\end{aligned}
$$

We set

$$
S(H)=\left\{m, s^{(1)}, \ldots, s^{(q)}, \ldots, s^{(t-1)}, s^{(t)}, \ldots, s^{(m-1)}=s_{\max }\right\}
$$

such that $f_{i}=s^{(i)}-m$ for any $i$ with $1 \leqq i \leqq q$ and

$$
P F^{*}(H)=\left\{f_{1}, \ldots, f_{q}, s^{(q+1)}-m, \ldots, s^{(t-1)}-m\right\} .
$$


We have

$$
\begin{aligned}
S(\tilde{H})= & \left\{2 m, 2 s^{(1)}, \ldots, 2 s^{(q)}, \ldots, 2 s^{(m-1)}\right\} \cup\left\{n, n+2 f_{1}, \ldots, n+2 f_{q}\right\} \\
& \cup\left\{n+2 s^{(q+1)}, \ldots, n+2 s^{(t-1)}, n+2 s^{(t)}, \ldots, n\right. \\
& \left.+2 s^{(m-2)}, n+2 s^{(m-1)}=n+2 s_{\max }\right\} .
\end{aligned}
$$

It follows from the assumption $n \geqq 2 c(H)+2 m-1$ that

$n+\left(n+2 s^{(j)}\right)>2 c(H)+2 m-2+n+2 s^{(j)}=2 s_{\text {max }}+n+2 s^{(j)}>n+2 s_{\text {max }}$.

Hence, we obtain

$$
P F(\tilde{H}) \supseteqq\left\{n+2 s^{(q+1)}, \ldots, n+2 s^{(t-1)}, n+2 s_{\max }\right\}
$$

Thus, we get $t(\tilde{H}) \geqq t-q$.

Combining Proposition 3 with Lemma 4 we can find non-Weierstrass numerical semigroups $\tilde{H}$ with an even conductor $c(\tilde{H})=2 g(\tilde{H})-2 u$ for any positive integer $u$.

Theorem 8 Let $v$ be a positive integer. Then there exists an infinite number of nonWeierstrass numerical semigroups $H$ with $c(H)=2 g(H)-2 v$.

Proof By Proposition 3 for any odd number $v \geqq 1$ we get an infinite number of nonWeierstrass numerical semigroups $H$ with $c(H)=2 g(H)-2 v$ and $t(H)=v+2$ because $l$ in Proposition 3 is any integer larger than 1 . We set $q=v-1$ in Lemma 4. Let $\tilde{H}$ be the numerical semigroup as in Lemma 4. Then it follows from Lemma 4 that

$$
c(\tilde{H})=2 g(\tilde{H})-2(v+v-(v-1))=2 g(\tilde{H})-2(v+1) .
$$

If $n \geqq 8 g\left(H_{0}\right)+2 v+1$, then we have

$$
\begin{aligned}
g(\tilde{H}) & =2 g\left(H_{0}\right)+\frac{n-1}{2}-q \geqq 2 g\left(H_{0}\right)+\frac{8 g\left(H_{0}\right)+2 v+1-1}{2}-q \\
& =6 g\left(H_{0}\right)+v-q=6 g\left(H_{0}\right)+1,
\end{aligned}
$$

which implies that $\tilde{H}$ is a non-Weierstrass numerical semigroup with $c(\tilde{H})=2 g(\tilde{H})-$ $2(v+1)$ by Remark 2, because $H=d_{2}(\tilde{H})$ is a non-Weierstrass numerical semigroup. Since $v$ is any odd number larger than or equal to 1 , any positive even numbers can be obtained by $v+1$.

Acknowledgements The author thanks the referee for his/her helpful comments, especially on the simplification of the proof of Proposition 1. 
Open Access This article is licensed under a Creative Commons Attribution 4.0 International License, which permits use, sharing, adaptation, distribution and reproduction in any medium or format, as long as you give appropriate credit to the original author(s) and the source, provide a link to the Creative Commons licence, and indicate if changes were made. The images or other third party material in this article are included in the article's Creative Commons licence, unless indicated otherwise in a credit line to the material. If material is not included in the article's Creative Commons licence and your intended use is not permitted by statutory regulation or exceeds the permitted use, you will need to obtain permission directly from the copyright holder. To view a copy of this licence, visit http://creativecommons.org/licenses/by/4.0/.

\section{References}

1. Buchweitz, R.O.: On Zariskis criterion for equisingularity and non-smoothable monomial curves. Preprint 113, University of Hannover (1980)

2. Hurwitz, A.: Über algebraischer Gebilde mit eindeutigen Transformationen in sich. Math. Ann. 41, 403-442 (1893)

3. Kato, T.: A simple proof for a result of Komeda-Ohbuchi, p. 5 (Unpublished manuscript)

4. Kim, S.J.: On the existence of Weierstrass gap sequences on trigonal curves. J. Pure Appl. Algebra 63, 171-180 (1990)

5. Komeda, J.: Cyclic coverings of an elliptic curve with two branch points and the gap sequences at the ramification points. Acta Arith. 81(3), 276-297 (1997)

6. Komeda, J.: Non-Weierstrass numerical semigroups. Semigroup Forum 57, 157-185 (1998)

7. Komeda, J.: Double coverings of curves and non-Weierstrass semigroup. Commun. Algebra 41, 312324 (2013)

8. Komeda, J., Ohbuchi, A.: On double coverings of a pointed non-singular curve with any Weierstrass semigroup. Tsukuba J. Math. Soc. 31, 205-215 (2007)

9. Komeda, J., Ohbuchi, A.: On $\gamma$-hyperelliptic Weierstrass semigroups of genus $6 \gamma+1$ and $6 \gamma$. Bull. Braz. Math. Soc. 48, 209-218 (2017)

10. Nari, H.: Symmetries on almost symmetric numerical semigroups. Semigroup Forum 86, 140-154 (2013)

11. Oliveira, G., Stöhr, K.-O.: Gorenstein curves with quasi-symmetric Weierstrass semigroups. Geom. Dedic. 67, 45-63 (1997)

12. Rosales, J.C., Garcia-Sánchez, P.A.: Numerical Semigroups, Developments in Mathematics, vol. 20. Springer, New York (2009)

13. Torres, F.: Weierstrass points and double coverings of curves with application: symmetric numerical semigroups which cannot be realized as Weierstrass semigroups. Manuscr. Math. 83, 39-58 (1994)

14. Torres, F.: On certain $N$-sheeted coverings of curves and numerical semigroups which cannot be realized as Weierstrass semigroups. Commun. Algebra 23, 4211-4228 (1995)

Publisher's Note Springer Nature remains neutral with regard to jurisdictional claims in published maps and institutional affiliations. 